\title{
Effects of Connected and Automated Vehicles in a Coopera.ve Environment
}

\author{
Ondrej Pribyl1 \\ ${ }^{1}$ Czech Technical University in Prague, Faculty of Transportation Sciences, Department of Applied Mathematics, Na Florenci 25, Praha 1, \\ 11000, Czech Republic. pribylo@fd.cvut.cz
}

\begin{abstract}
Cooperative and automated vehicles (CAVs) are often considered a mean to improve quality of life in cities, the traffic flow parameters in particular. This paper provides some evidence based on microscopic traffic simulation on how the effects can really be. Important is that the particular use cases are not built in vehicles only. We focus on so called cooperative environment and advanced traffic control measures.

This paper describes the impact of CAVs on a cooperative urban environment, resulting from a European research project MAVEN. We clearly demonstrate that a proper integration of CAVs into city traffic management can, for example, help with respect to the environmental goals and reduce CO2 emissions by up to $12 \%$ (a combination of GLOSA and signal optimization). On corridors with a green wave, a capacity increase of up to $34 \%$ was achieved. Already for lower penetration rates (20\% penetration of CAVs), there are significant improvements in traffic performance. For example, platooning leads to a decrease of $\mathrm{CO} 2$ emissions of $2,6 \%$ or an impact indicator by $17,7 \%$.
\end{abstract}

Keywords: Traffic management, Smart Solutions for safe, Efficient and sustainable traffic flow

JEL: R410 Transportation: Demand, Supply, and Congestion; Travel Time; Safety and Accidents; Transportation Noise

\section{Introduction}

Cooperative and automated vehicles (CAVs) are often considered a mean to improve quality of life in cities. CAVs do not only serve as a new source of information (for example to estimate the queue-length at an intersection with higher precision), but through for example speed or lane change advisory or routing algorithms, they can make traffic more energy-efficient and fluent, and the traffic flow in the network more balanced. So much the expectations. But what is the real effect of vehicle automation? Before we answer this question, let us provide some definitions. The terminology in the field is not unified. The particular letters in the abbreviation CAV can gave different meaning. There is for example a big difference in the usage in Europe and in the USA.
For example, the letter " $C$ " in the abbreviation can mean cooperative or connected, the letter " $A$ " then automated or autonomous. Within this paper we adopt terminology from Andata (https://www.andata.at/en/answer/whats-the-differencebetween-autonomous-automated-connected-andcooperative-driving.html):

Automated vehicles denote self-driving vehicles, i.e. vehicles that can drive without human intervention. Autonomous vehicles describe vehicles that are allowed to make decisions independently and on one's own mind. Further, different levels of automation are defined for example by SEA international (https://www.sae.org/news/ 2019/01/sae-updates-j3016-automated-driving-graphic) and provided in Fig. 1. 


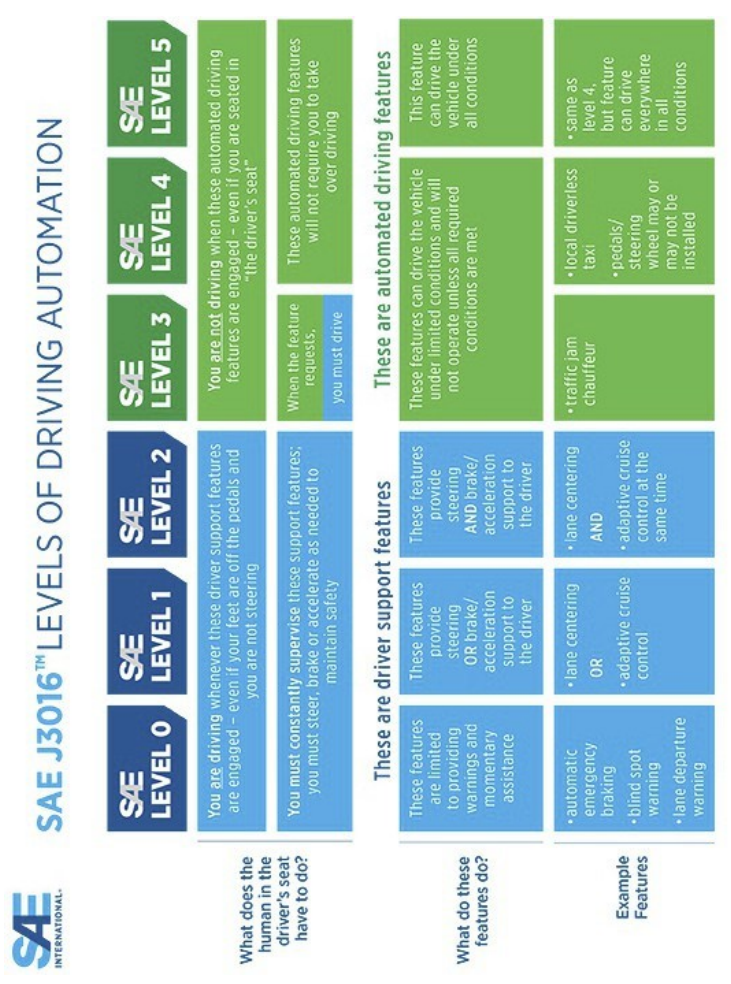

Figure 1: Levels of automation according to SAE (source: SAE International: https://www.sae.org/news/2019/01/saeupdates-j3016-automated-driving-graphic)

Connected vehicles are exchanging information among each other and/or infrastructure in an automated way.

Coopera.ve vehicles act cooperatively within traffic, which implies that they are coordinating their microscopic aims and actions in the light of improved overall macro-scopic effects.

Within this paper, the abbreviation CAV denotes cooperative and automated vehicles. We focus on automation level 4 and 5 .

Now let us get back to the question whether cooperativeness and automation will have a positive or negative effect on traffic and environmental parameters. The early expectations were really optimistic. It was expected that autonomous vehicles will improve safety, reduce congestions, harmonize traffic, reduce the number of vehicles on roads, allow for savings in infrastructure incl. parking or for example make more affordable mobility services.

In the last years, however, researchers started to doubt the expected positive impacts and often refer back to them as "wishful thinking". Even when the challenges from above are overcome, the impact on, for example, traffic flow can be really different. At best, there is much uncertainty about the impact of AVs as it is demonstrated in the following paragraphs [1].

Sousa et al. [2] stated that since automated vehicles can provide mobility for new groups of users, travel demand may increase.

This statement was supported by the work of Sivak and Schoettle [3], who analysed the reasons for not having a driving license and estimated this increase in new mobility users to go as high as $11 \%$ when AVs become widely accessible. Harper et al. [4] used the data from the $2009 \mathrm{Na}$ tional Household Transportation Survey to study this phenomenon. His results suggest that in the USA, the increase in vehicle miles travelled is expected to be $14 \%$.

The cities and the entire land use will need to be changed to address another important aspect of autonomous vehicles. Nowadays, cars in cities are used only for short periods of time. People typically drive to work in the morning and back home in the late afternoon. In the meantime, they stay parked.

Finding a parking spot may, in addition to fuel and time waste and increase in the overall stress, increase traffic up to $15 \%$ [5].

AVs address these problems by driving passengers to their destination, and then driving to a dedicated parking place at home or outside of the city centre. This can reduce the need for parking places in the centre, but introduces new challenges. The empty AVs would be negatively influencing the overall traffic flow, using extra fuel and polluting while looking for parking far away [6].

Additionally, the city would need changes in the entire land use, for example, an additional space and solution for drop-off and pick up by AVs [7].

Automated vehicles should save space not only by reducing the number of parked vehicles but also by reducing the space required for parking them. AVs allow parking in so-called depots where the space needed to park such vehicles can be reduced to half the space needed with conventional parking lot designs [8].

David Metz [9] confirms the expectations that the impact of AVs cannot be simply just postive or just negative. He addresses the concept of autonomous driving with respect 
to other new trends - the topic of vehicle ownership and ride-sharing. He puts together the partial conclusions from previous sections and concludes that it is to be expected that individually owned AVs will add significantly to the overall distance travelled by car and hence to increased traffic levels. In contrast, AVs operating as robotic taxis would not be expected to have such an impact, given that conventional taxis travel without passengers between paid trips.

Additionally, the lower travel costs associated with using shared vehicles can attract passengers from public transport, increasing the demand for a private car or taxi use.

This effect was also in details elaborated by Wadud et al [10]. The authors explore the effects of automation on congestions, energy consumption and emissions through several illustrative scenarios, finding that automation might plausibly reduce road transport emissions and energy use by nearly half; or nearly double them; depending on which effects come to dominate.

A critical question is whether autonomous vehicles increase or reduce total vehicle travel and associated external costs. It could go either way, depending on public policies. By increasing travel convenience and comfort, and allowing vehicle travel by non-drivers, they could increase total vehicle mileage, but they may also facilitate vehicle sharing, which allows households to reduce vehicle ownership and therefore total driving.

\section{Cooperative Environment}

The previous section provided evidence from existing literature that just the fact you have automated vehicles in cities does not automatically mean an improvement in traffic parameters. Apart from the adopted policies also the way in which they are integrated into the existing traffic management are crucial. Just that a vehicle does not have a driver does not lead to decrease in travel time.

Within this paper we would like to enhance the definitions from above and define so-called:

Cooperative environment - where not only a single traffic participant is automated and exchanging information, but the infrastructure also plays an important role by providing additional functionality and higher efficiency of its

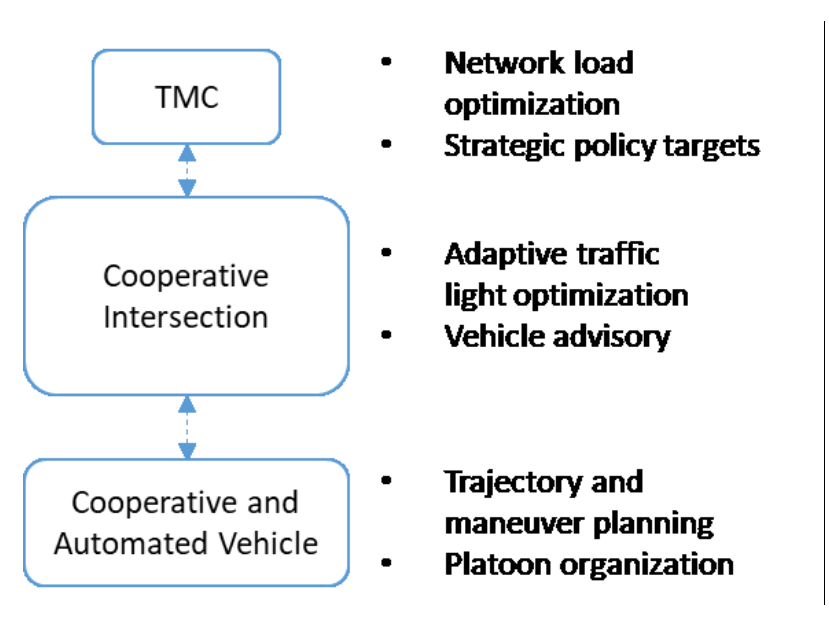

Figure 2: Focus of the use cases defined in the MAVEN project

algorithms.

It must be mentioned we are not necessarily talking only about new functionality, but the existing must be newly defined and improved with the new information it gets from cooperative vehicles. This is true for example for queue length estimation algorithms. Newly, they get data not only from the static traffic sensors, but also from the cooperative vehicles. The precision of such algorithms can be significantly improved, especially for higher penetration rates of automate cooperative vehicles.

The scope and focus of the project MAVEN defined above is provided in Fig. 2. The algorithms are not located on one particular point, but are distributed among the traffic management centers and intersections on the infrastructural part, and among particular cooperative vehicles in the network. For example, so called cooperative perception means, that a vehicle gets information about possible object either from its own sensors, from sensors of another vehicle or even from sensors (e.g. hemispherical cameras) located at the infrastructure. In such was, it is not limited to its own field of view but can make decisions based on more data. This is though related also the concept of "data security" and "trust" and is thus suitable for application of so-called multiagent systems.

Within the project maven, several use cases were implemented and evaluated. We are not going to describe the particular algorithms here, as they are described in [11] in details. Here we provide just an overview of the use cases (UC) and methods implemented.

Platooning (UC1-6) A promising possibility to enhance 
future traffic efficiency is the formation of platoons by automated vehicles. For simplicity, in the traffic simulation model, we regard a platoon as a group of automated vehicles following each other with a reduced time headway and possibly employing additional control schemes to maintain a coherent state within the group.

GLOSA (UC7) - Green Light Optimal Speed Advisory The main concept of this algorithm is that vehicles receive SPaT messages containing switching times of the next traffic light. An on-board assistance system in the vehicle computes an optimal approaching speed so that the vehicle can pass the traffic light without stopping.

Lane Change Advisory (UC8)- This feature of automated vehicles allows the exchange of information between the approaching vehicle and the C-ITS infrastructure on the intersection in order to inform the vehicle about the queue lengths on different lanes of the intersection approach. By mutual exchange between vehicle and infrastructure, precise queue length can be estimated, and thus vehicles can be redirected to less congested lanes of the approach.

Signal optimization (UC 14) is a feature that brings the benefits for traffic management and is specifically designed to support GLOSA for automated vehicles. Existing adaptive traffic light control algorithms, can adapt more efficiently and accurately thanks to enhanced information source CAV of UC 11 and the GLOSA algorithm itself also uses the enhanced queue information. Applied signal control algorithm has the potential to bring benefits even without the utilization of CAV information. In order to depict these benefits, we introduce to the graphs in this section not only data from simulations with different CAV penetration rate but also a baseline scenario data reflecting the current control algorithms.

Network coordination / Green wave (UC15) is a wellknown phenomenon described for example in D4.4 [4]. The literature describes this Use Case as coordination of signal phases on intersections in such a manner, as to provide coordinated waves of green lights on the intersections that are positioned on a main traffic flow trajectory over the network.

Combined use cases (UC ALL) In order to demonstrate the joint impact of the particular use cases the effectiveness and impact on traffic flow of all Use Cases that can be combined on the signal intersection network of Helmond, i.e. the platooning (UC1-6), GLOSA (UC7), Lane change advisory (UC8), network coordination (UC13) and signal optimization (UC14).

\section{Simulation Environment}

In order to ensure the validity of the results, each of the performed simulations was thoughtfully planned, analyzed and calibrated in order to minimize the discrepancies from the realworld behaviour of vehicles in a baseline simulation. The baseline scenario was calibrated using realworld data collected in particular networks. Each simulated scenario with tested UC was performed 10 times for each parameter setting, and the results were averaged to ensure a statistically significant outcome. The equation to compute the exact number of required simulation runs is provided for example in [54]. This is important to ensure that achieved results are not overwhelmed by stochastic discrepancies.

It is important to note, that Use Cases 1-6 and 15, which are platooning of the CAV vehicles and Negotiation between CAV and C-ITS is implemented in all of the simulations. Therefore, their indirect impact on the simulations is tested in each of the simulated scenarios. To implement the algorithms necessary for testing the full range of planned UC, a corridor with multiple intersections in Helmond was built up using SUMO, shown in Figure 36. Seven intersections: intersection 701, 702, 704, 101, 102, 103 and 104 are distributed on this stretch of corridor, with the same main direction, east-west through directions 2 and direction 8 for each aforementioned intersection. Respectively, signal group 2 of each intersection is eastwest bound and signal group 8 is west-east bound. The configuration of the two signal groups, SG 2 and SG 8 of intersection 701 are almost identical. They contain the same number of lanes (two lanes each), have the same saturation flow (1800 vehicle/hour), the same number of signal heads and they both appear in the same stages/ stage assignment. The simulated traffic is detected in SUMO, then the detected vehicle information is sent back to ImFlow to calculate and optimize the signal timing plan. After making the decision of which plan to choose, ImFlow sends back the chosen plan to SUMO to continue the 
simulation. The detection type of SG 2 and SG 8 are both set to adaptive unconditional in ImFlow configurator. Therefore, stabilized GLOSA can be provided to these two signal groups. More detailed configuration of the simulation in Helmond network was provided in deliverable [11].

\section{Selected Simulation Results}

The following sections describe just some selected findings of the project MAVEN. For more details and further figures, please refer to [12].

\subsection{Effects of Platooning}

Let us look at the effects of the vehicle platooning on average delay. Fig. 3 depicts the average delay in seconds ( $y$ axis) for the increasing ratio of CAV vehicles ( $x$-axis) and nominal as well as $60 \%$ traffic volume. There is a significant decrease in the average delay, let us look in details on the nominal traffic volume. An especially large reduction of $23 \%$ can be seen with the first introduction of $20 \%$ ratio of CAV penetration (10runp20). With the assumption of $100 \%$ penetration rate of CAVs in the traffic flow, the resulting average delay decreases by $52 \%$.

In general, the highest expected improvements in almost all aspects (i.e. impact, emissions and queue lengths) happens at the penetration levels of $20 \%$. For example, the queue lengths decrease by about $20 \%$ for this penetration rate, while in case of automated vehicles only (penetration level of $100 \%$ ) is the decrease about $39 \%$ in queue length. This is an important conclusion looking at the transition phase (i.e. mixed traffic): significant effects can be

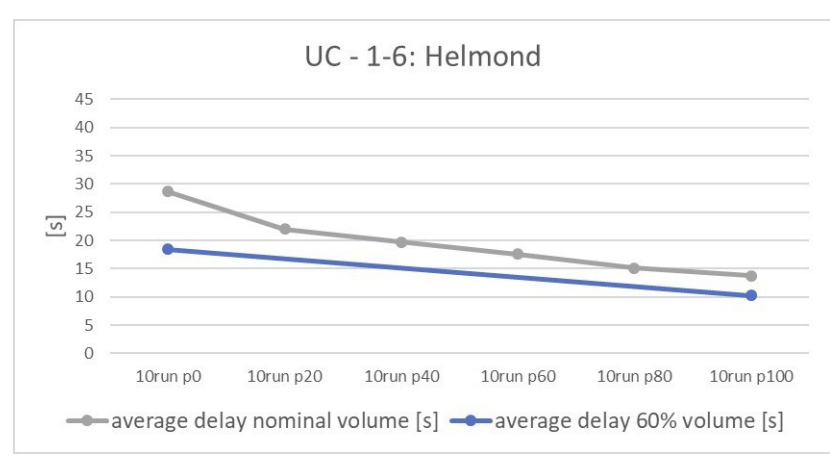

Figure 3: Effect of platooning on average delay expected at an early stage.

For the full penetration of automated vehicles, the impact on $\mathrm{CO} 2$ emissions reaches over $8 \%$, which is a significant improvement.

\subsection{Effects of Speed Change Advice and Green Wave Optimization}

An important use case combines the green wave optimization (i.e. network coordination) with the speed change advice, so that the vehicles arrive at the end of starting queue. For the nominal traffic volume and $60 \%$ penetration of CAVs, there is a decrease in $\mathrm{CO} 2$ emissions of about $5,4 \%$.

Especially for the penetration level of automated vehicles equal to $60 \%$, there is a significant improvement in the average number of stops of about $48,4 \%$, or average queue lengths of about $50,7 \%$ (see Table 1). For the penetration level of automated vehicles equal to $100 \%$, there is an

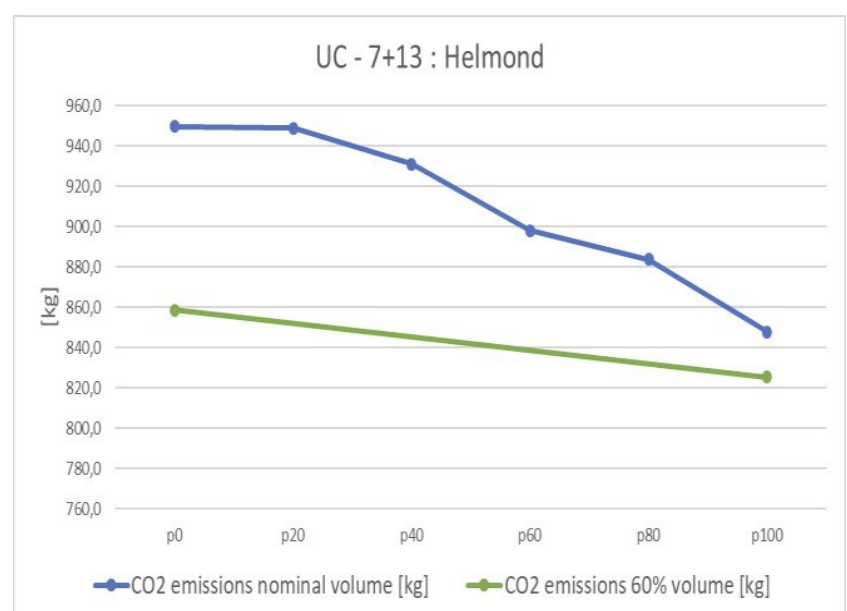

Figure 4: Effect of Speed change advice and Green wave optimization on $\mathrm{CO} 2$ emissions

\begin{tabular}{|c|c|c|c|c|}
\hline $\begin{array}{c}\text { Penetration } \\
\text { rate }\end{array}$ & $\begin{array}{c}\text { Average } \\
\text { delay [s] }\end{array}$ & $\begin{array}{c}\text { Average } \\
\text { mumber of } \\
\text { stops [-] }\end{array}$ & $\begin{array}{c}\text { Average } \\
\text { queue } \\
\text { length [m] }\end{array}$ & $\begin{array}{c}\mathrm{CO}_{2} \\
\text { emissions } \\
{[\mathrm{kg}]}\end{array}$ \\
\hline $\mathrm{p} 0$ & 40,8 & 0,9 & 13.2 & 949.7 \\
\hline $\mathrm{p} 20$ & 36,2 & 0,8 & 12,7 & 948,9 \\
\hline $\mathrm{p} 40$ & 32,0 & 0,7 & 10,4 & 931,1 \\
\hline $\mathrm{p} 60$ & 24,5 & 0,5 & 6,5 & 898,1 \\
\hline $\mathrm{p} 80$ & 21,8 & 0,4 & 5,6 & 883,7 \\
\hline $\mathrm{p} 100$ & 17,9 & 0,3 & 3,4 & 847,8 \\
\hline
\end{tabular}

Table 1: Overview of benefits of UC $13+7$ and nominal traffic volume 
expected reduction of emissions of about 10\%. Specific simulations isolated the capacity and revealed an increase of $34 \%$ thanks to the combined use cases.

\subsection{Effects of Speed change advice and Signal optimiza- tion}

Signal optimization is a feature that brings the benefits for traffic management and is specifically designed to support GLOSA for automated vehicles. Existing adaptive traffic light control algorithms, can adapt more efficiently and accurately thanks to enhanced information source. The first and most anticipated benefit of signal optimization through cooperation with CAV is the reduction of average delay over all network. This expectation seems to be met by results achieved in Helmond network depicted in Fig. 3. The vertical grey line depicts also the baseline scenario without the signal optimization algorithm. You can see, that even for a $0 \%$ penetration lane (i.e. network without automated vehicles), there is already an improvement in delay of about $6.4 \%$. Compared to this baseline value, the algorithm with a $100 \%$ penetration rate of CAVs can improve the delay by over $43 \%$. This combination clearly improved the measured indicators. This is true for low demand as well as the nominal traffic volume. For example, we achieved reduction of $\mathrm{CO} 2$ emissions for the full penetration of automated vehicles was about 5\%.

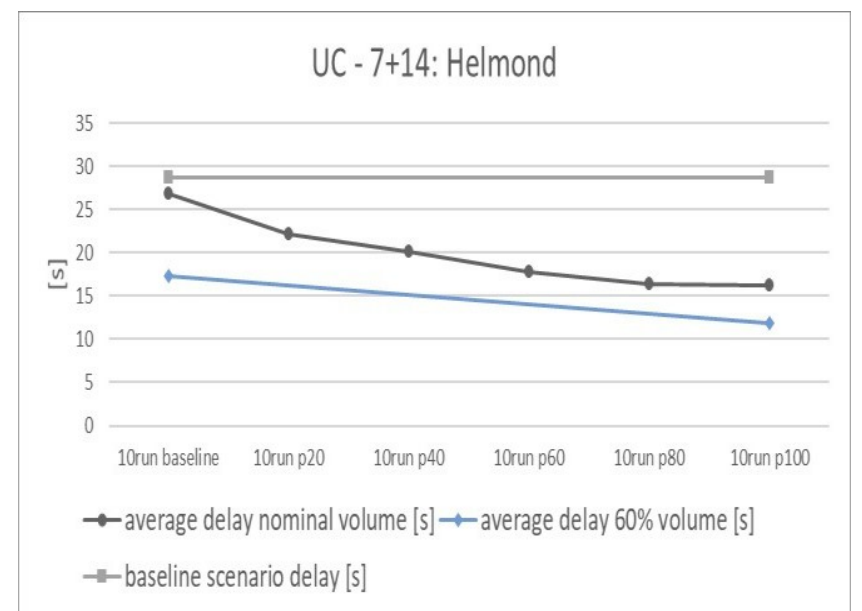

Figure 5: Effect of Speed change advice and Signal optimization on average delay

\subsection{Effects of all combined use cases}

In order to demonstrate the joint impact of the particular MAVEN Use cases, the following section examines the effectiveness and impact on traffic flow of all Use Cases that can be combined on the signal intersection network of Helmond, i.e. the platooning (UC1-6), GLOSA (UC7), Lane change advisory (UC8), Queue modelling (UC11), signal optimization (UC14) and negotiation (UC15). Here only the nominal traffic volume is evaluated.

The combination of all use cases has a very positive effect on the average delay, queue length, and impact. For example, already for $20 \%$ penetration rate of autonomous vehicles, there is a decrease of average delay by about $23 \%$. For a penetration rate of $100 \%$, there is a decrease of over $52 \%$ (Fig. 6). On the other hand, it has a negative impact on the number of stops (Fig. 7) and CO2 emissions. This indicates that it might be advisable to apply the different use cases carefully for each traffic and network situation and also with respect to the expected impact on traffic. Minimizing the delay does not necessarily lead to most harmonized traffic flow.

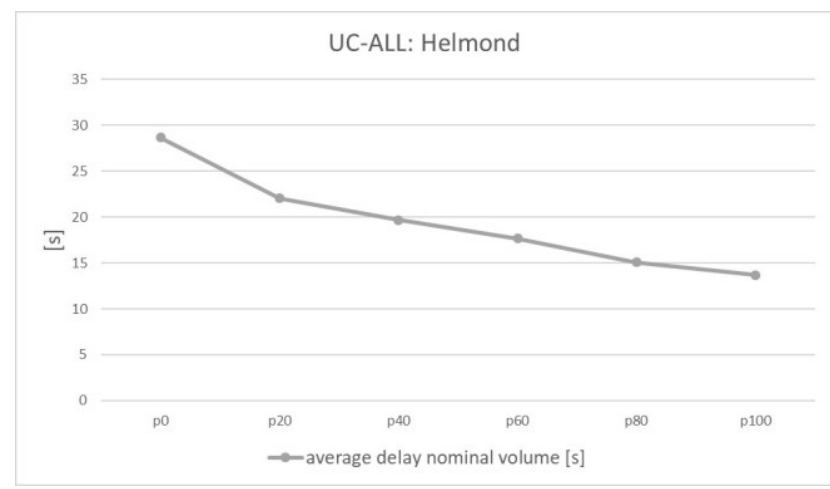

Figure 6: Effect of the combination of all use cases on the average delay

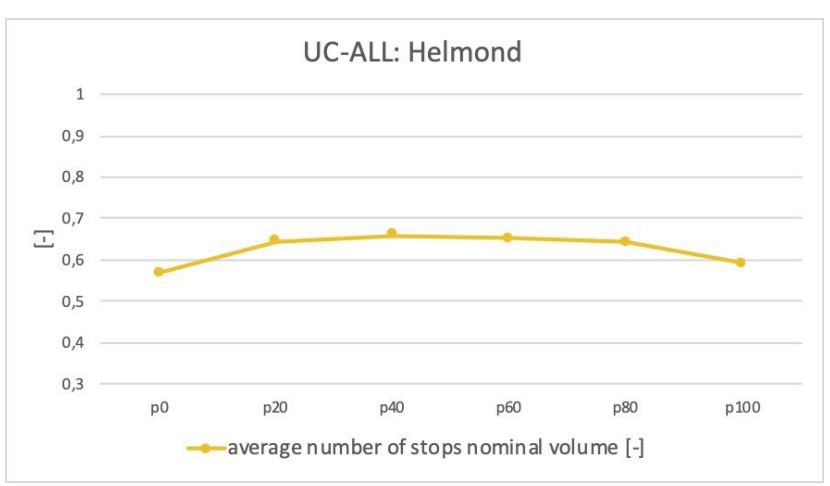

Figure 7: Effect of the combination of all use cases on number of stops 


\section{Conclusions}

Cooperative and automated vehicles (CAVs) are often considered a mean to improve quality of life in cities. CAVs do not only serve as a new source of information (for example to estimate the queue-length at an intersection with higher precision), but through for example speed or lane change advisory or routing algorithms, they can make traffic more energy-efficient and fluent, and the traffic flow in the network more balanced.

This paper describes the impact of CAVs on a cooperative urban environment, resulting from a European research project - MAVEN. The evaluation covered different dimensions, including user surveys, field tests and simulations. Here, the results from a microscopic traffic simulation (using tool SUMO) demonstrating the impact on different indicators (such as number of stops, delay or emissions) are presented. One of the key advantages of this approach is in addressing the effects not only for different layouts or traffic volumes, but also for the transition phase, i.e. different penetration rates of CAVs.

We clearly demonstrate that a proper integration of CAVs into city traffic management can, for example, help with respect to the environmental goals and reduce $\mathrm{CO} 2$ emissions by up to $12 \%$ (a combination of GLOSA and signal optimization). On corridors with a green wave, a capacity increase of up to $34 \%$ was achieved. Already for lower penetration rates (20\% penetration of CAVs), there are significant improvements in traffic performance. For example, platooning leads to a decrease of $\mathrm{CO} 2$ emissions of $2.6 \%$ or an impact indicator by $17.7 \%$.

The real impact of CAVs however depends not only on the algorithms used, but also on policies adopted. Some use cases can be aiming on contradictory performance indicators and it is crucial that traffic managers understand the big picture and integrate policies enabled by automation (car sharing, electro-mobility, and others). The findings can be used not only by other researchers but mainly by traffic managers and decision-makers in cities, as they can provide a better idea about the actual impacts of particular solutions in a cooperative environment and to help with the transition phase.

\section{ACKNOWLEDGEMENTS}

This research was funded by the European Horizon 2020 Program, MAVEN project [Grant agreement no: 690727].

\section{REFERENCES}

Ross, C. and Guhathakurta, S. Autonomous Vehicles and Energy Impacts: A Scenario Analysis, Energy Procedia, Volume 143, 2017, Pages 47-52. Sousa N, Almeida A, Coutinho-Rodrigues J and Nativida-de-Jesus E (2017) Dawn of autonomous vehicles: review and challenges ahead. Proceedings of the Institution of Civil Engineers Municipal Engineer 171(1): 3-14.

Sivak M and Schoettle B (2015) Influence of Current Non-drivers on the Amount of Travel and Trip Patterns with Self-Driving Vehicles. The University of Michigan Transportation Research Institute, Ann Arbor, MI, USA. UMTRI-2015-39. Harper C, Hendrickson C, Mangones S and Samaras C (2016) Estimating potential increases in travel with autonomous vehicles for the nondriving, elderly and people with travel-restrictive medical conditions. Transportation Research Part C: Emerging Technologies 72: 1-9

5] Alessandrini A, Campagna A, Site P, Filippi F and Persia L (2015) Automated vehicles and the rethinking of mobility and cities.

Transportation Research Procedia 5: 145-160 Ambühl, L., F. Ciari and M. Menendez (2016) What about space? A simulation based assessment of AVs impact on road space in urban areas, 16th Swiss Transport Research Conference, Ascona, Switzerland, May 2016.

Heinrichs D. (2016) Autonomous Driving and Urban Land Use. In: Maurer M., Gerdes J., Lenz B., Winner H. (eds) Autonomous Driving. Springer, Berlin, Heidelberg

Ferreira, M., Damas, L., Conceição, H., d'Orey, P.M., Fernandes, R., Steenkiste, P., \& Gomes, P. (2014). Self-automated parking lots for autonomous vehicles based on vehicular ad hoc networking. 2014 IEEE Intelligent Vehicles Symposium Proceedings, 472-479. 
Journal for Mobility and Transport

[9] Metz, D. 2018. Developing Policy for Urban Autonomous Vehicles: Impact on Congestion. Urban Science. MDPI, Vol. 2/33

[10] Wadud, Z., MacKenzie, D. and Leiby, P. (2016). Help or hindrance? The travel, energy and carbon impacts of highly automated vehicles.

Transportation Research Part A: Policy and Practice 86, 1-18.

[11] Blokpoel, R. et al. 2018. MAVEN Project - Managing Automated Vehicles enhances Network. Deliverable No D4.4 - Cooperative adaptive traffic light with automated vehicles. Technical report. p. 116

[12] Pribyl, O. et al. 2019.MAVEN Project - Managing607Automated Vehicles Enhances Network. Deliverable No D7.2 - Impact Assessment, Technical Report. p. 168 